\title{
The R-SNARE Endobrevin/VAMP-8 Mediates Homotypic Fusion of Early Endosomes and Late Endosomes
}

\author{
Wolfram Antonin, ${ }^{*+}$ Claudia Holroyd, ${ }^{*+}$ Ritva Tikkanen, ${ }^{\ddagger}$ Stefan Höning, ${ }^{\S}$ \\ and Reinhard Jahn ${ }^{+\|}$
}

\author{
${ }^{+}$Department of Neurobiology, Max-Planck-Institute for Biophysical Chemistry, Göttingen, Germany; \\ łInstitute for Cell Biology, University of Bonn, Bonn, Germany; and SInstitute for Biochemistry II, \\ University of Göttingen, Göttingen, Germany
}

Submitted January 12, 2000; Revised April 24, 2000; Accepted July 27, 2000

Monitoring Editor: Suzanne R. Pfeffer

\begin{abstract}
Endobrevin/VAMP-8 is an R-SNARE localized to endosomes, but it is unknown in which intracellular fusion step it operates. Using subcellular fractionation and quantitative immunogold electron microscopy, we found that endobrevin/VAMP-8 is present on all membranes known to communicate with early endosomes, including the plasma membrane, clathrin-coated pits, late endosomes, and membranes of the trans-Golgi network. Affinity-purified antibodies that block the ability of endobrevin/VAMP-8 to form SNARE core complexes potently inhibit homotypic fusion of both early and late endosomes in vitro. Fab fragments were as active as intact immunoglobulin Gs. Recombinant endobrevin/VAMP-8 inhibited both fusion reactions with similar potency. We conclude that endobrevin/VAMP-8 operates as an R-SNARE in the homotypic fusion of early and late endosomes.
\end{abstract}

\section{INTRODUCTION}

Trafficking of intracellular membranes involves the fusion of vesicles with their respective target membranes. Intracellular fusion events are mediated by complementary sets of SNARE proteins that are localized on the membranes destined to fuse (Rothman, 1994). SNAREs constitute a superfamily of proteins that share a common motif (the SNARE motif of $\sim 60-70$ amino acids) (Jahn and Südhof, 1999). The best characterized SNAREs are those functioning in exocytosis of synaptic vesicles. They include the vesicle protein synaptobrevin (also referred to as VAMP) and the plasma membrane proteins syntaxin 1 and SNAP-25. These proteins assemble spontaneously into a ternary complex that is disassembled by the chaperone ATPase NSF (NEM [N-ethylmaleimide]-sensitive factor) in conjunction with cofactors called SNAPs (soluble NSF attachment proteins) (Söllner et al., 1993). According to a current model, the assembly drives the fusion reaction by forming a tight connection between the SNAREs in the partner membranes (trans complexes).

\footnotetext{
* These authors contributed equally to this work.

\| Corresponding author. E-mail address: rjahn@gwdg.de. Abbreviations used: BSA-gold, BSA coupled to 5-nm gold; ER, endoplasmic reticulum; NSF, NEM [N-ethylmaleimide]-sensitive factor; SNAP, soluble NSF attachment protein; SNARE, SNAP receptor; TGN, trans-Golgi network; VAMP, vesicle-associated membrane protein.
}

After fusion, the SNAREs within the complex are all aligned in parallel (cis complexes). They then need to be reenergized for another round of fusion by NSF and ATP-mediated disassembly (Hanson et al., 1997).

Sequence comparison revealed that all known SNARE motifs fall into two major subfamilies that contain either a conserved glutamine (Q-SNAREs) or a conserved arginine (R-SNAREs) at a central position (Fasshauer et al., 1998b; Weimbs et al., 1998). In the neuronal SNARE complex, three Q-SNARE motifs (one contributed by syntaxin and two by SNAP-25) and one R-SNARE motif (contributed by synaptobrevin/VAMP) form an extended helical bundle (Sutton et al., 1998). The glutamines and the arginine interact to form an ionic layer in the middle of the helical bundle that is surrounded by less well conserved hydrophobic layers. A similar composition of three Q-SNAREs and one R-SNARE was also found in a corresponding SNARE complex of yeast. Together, these observations suggest that all SNARE complexes consist of such four-helix bundles (three Q-SNARE motifs, one R-SNARE motif) with an asymmetric ionic layer in the middle.

SNARE complexes other than those functioning in exocytosis of neurons and yeast, however, are less well characterized. For instance, in yeast the homotypic fusion of vacuole precursors is probably mediated by the SNAREs Nyv1p, Vam7p, and Vam3p (Nichols et al., 1997; Ungermann and Wickner, 1998), and possibly also Vti1p (Götte and von Mollard, 1998). Similarly, the SNAREs Sed5p, Sec22p, Bet1p, 
and Bos1p are candidates for the fusion of endoplasmic reticulum (ER)-derived trafficking vesicles with the cisGolgi, but some of these proteins may also be involved in retrograde traffic from the cis-Golgi to the ER (Spang and Schekman, 1998). In mammalian cells, the Sed5 orthologue syntaxin 5 has been shown to be required for the fusion of ER-derived trafficking vesicles (Dascher et al., 1994; Dascher and Balch, 1996) with the Golgi apparatus as well as for the reassembly of Golgi stacks after mitosis (Rabouille et al., 1998).

Recently, many novel SNAREs have been identified, mostly as a result of the rapid growth of expressed sequence tag databases (Bock and Scheller, 1997). Most of them appear to be localized to specific subsets of intracellular membranes, suggesting that they specifically mediate distinct fusion steps (for review, see Jahn and Südhof, 1999). However, a precise subcellular localization of a given SNARE is a prerequisite, but it is by no means sufficient to pinpoint the fusion step in which it functions. Every trafficking vesicle derived from a donor compartment carries SNAREs required for fusion with its target membrane. Because after fusion the SNAREs need to recycle to the donor compartment, they must be present not only in the donor and target compartments but also in all intermediates involved in the recycling pathway and, furthermore, in the membranes involved in its biogenesis. It is essential, therefore, to map a given SNARE precisely on intracellular recycling pathways before hypotheses about the fusion step it mediates can be proposed.

In the present study, we have focused on the role of a recently discovered R-SNARE, endobrevin/VAMP-8 (Advani et al., 1998; Wong et al., 1998b). Endobrevin is only distantly related to the synaptobrevins and appears to be localized mainly to an early endosomal compartment. We now report that in addition to early endosomes, endobrevin is present on late endosomes and the trans-Golgi network (TGN) as well as on coated pits and the plasma membrane, suggesting that it recycles by means of two distinct pathways. In line with this intracellular distribution, our data document that endobrevin functions as an R-SNARE in the homotypic fusion of both early and late endosomes.

\section{MATERIALS AND METHODS}

\section{Materials}

Rat syntaxin 1A (residues 1-265) in a pET22b vector encoding for a factor Xa-cleavable C-terminal $\mathrm{His}_{6}$ fusion protein and a thrombincleavable GST-endobrevin (residues 1-74) expression construct have been described (Fasshauer et al., 1999). The cDNAs for rat syntaxin 1A and SNAP-25 were kindly provided by R.H. Scheller (Stanford University of Medicine, Stanford, CA) and T.C. Südhof (UT Southwestern Medical Centre, Dallas, TX), respectively.

\section{Antibodies}

The following antibodies were described previously: synaptophysin (rabbit antiserum G96) (Jahn et al., 1985); cellubrevin (rabbit antiserum R54) (Annaert et al., 1997); endobrevin (rabbit antiserum) (Fasshauer et al., 1999); rab5 (mAb Cl 621.3) (Fischer von Mollard et al., 1994); and rab5 (rabbit antiserum R6) (Fischer von Mollard et al., 1994). The following antibodies were kind gifts: syntaxin (mAb HPC-1; provided by Dr. C. Barnstable, Yale School of Medicine, New Haven, CT) (Barnstable et al., 1985); Sec61 $\alpha$ (rabbit serum; provided by Dr. E. Hartmann, University of Göttingen, Göttingen,
Germany) (Görlich et al., 1992); LIMP II (rabbit antiserum and mAb provided by Y. Tenaka, Kyushu University, Fukuoka, Japan) (Barriocanal et al., 1986); and MPR46 (rabbit serum MSC1; provided by A. Hille-Rehfeld, University of Göttingen, Göttingen, Germany). SCAMP (rabbit serum) was obtained from Synaptic Systems (Göttingen, Germany).

\section{Preparation of Fab Fragments}

Synaptophysin antiserum was affinity purified as described (Navone et al., 1986). Endobrevin antiserum was affinity purified with the use of recombinant GST-endobrevin (Fasshauer et al., 1999) coupled to cyanogen bromide-Sepharose 4B (Pharmacia, Piscataway, NJ).

Affinity-purified antibodies against endobrevin and synaptophysin were digested with the use of papain beads (Sigma Chemical, St. Louis, MO) for $90 \mathrm{~min}$ at $37^{\circ} \mathrm{C}$ in PBS containing $1 \mathrm{mM}$ EDTA and $10 \mathrm{mM}$ cysteine, $\mathrm{pH}$ 7.4. The beads were then pelleted at $14,000 \times g$ for $5 \mathrm{~min}$. Antipain and PMSF were added to the supernatant at final concentrations of $2 \mu \mathrm{g} / \mathrm{ml}$ and $0.5 \mu \mathrm{M}$, respectively. Fab fragments were purified by ion exchange chromatography with the use of a Mono- $Q$ column on a fast-performance liquid chromatography system (Pharmacia). Fractions were tested by SDS-PAGE/immunoblotting for the presence of Fab fragments and undigested immunoglobulin $(\mathrm{Ig}) \mathrm{Gs}$. The Fab fragment containing fractions were free of IgGs, IgMs, or digestive products. The purified Fab fragments exhibited an affinity similar to that of undigested IgGs when tested by immunoblotting in serial dilutions.

\section{Subfractionation and Immunoisolation of Endosomes}

For separation of early and late endosomes, postnuclear supernatants (PNS) were fractionated with the use of isopycnic sucrose density gradient centrifugation. PNS were adjusted to $42 \%$ sucrose and overlaid with a discontinuous sucrose gradient according to Aniento et al. (1993). When the separation was performed after the fusion reaction, the incubation mix was loaded directly on top of a continuous sucrose gradient $(10-40 \%$ [wt/vol] dissolved in $3 \mathrm{mM}$ imidazole, $\mathrm{pH} 7.4,0.5 \mathrm{mM}$ EDTA, $1 \mu \mathrm{g} / \mathrm{ml}$ biotinylated insulin [Sigma; added as quencher]) and centrifuged for $19 \mathrm{~h}$ at 40,000 rpm in a Beckman (Fullerton, CA) SW 41 rotor.

For immunoisolation, mAb Cl 621.3 (anti-Rab5) and affinity-purified polyclonal anti-endobrevin antibodies were covalently coupled to Eupergit C1Z methacrylate microbeads as described (Burger et al., 1989). Liver was homogenized in homogenization buffer (320 $\mathrm{mM}$ sucrose, $5 \mathrm{mM}$ HEPES, pH 7.4, $1 \mathrm{mM}$ EDTA, $0.1 \mathrm{mM}$ GTP $\gamma$, and the following protease inhibitors: $10 \mu \mathrm{g} / \mathrm{ml}$ soybean trypsin inhibitor, $1 \mu \mathrm{g} / \mathrm{ml}$ pepstatin, $11 \mu \mathrm{g} / \mathrm{ml}$ benzamidine, $1 \mu \mathrm{g} / \mathrm{ml}$ antipain, $1 \mu \mathrm{g} / \mathrm{ml}$ leupeptin, $0.1 \mathrm{mM} \mathrm{PMSF}$ ) with the use of a glass-Teflon homogenizer (five strokes, $600 \mathrm{rpm}$ ). PNS was generated by centrifugation at $1000 \times g$ for $10 \mathrm{~min}$. PNS $(200 \mu \mathrm{g}$ of protein) was incubated in $400 \mu \mathrm{l}$ of homogenization buffer with 20 $\mu \mathrm{l}$ of the appropriate beads for $1 \mathrm{~h}$ at $4^{\circ} \mathrm{C}$. The incubation mixture was layered on top of a sucrose cushion $(0.5 \mathrm{ml}, 0.8 \mathrm{M})$ and centrifuged for $5 \mathrm{~min}$ at $4600 \times g$. The supernatants were centrifuged for $30 \mathrm{~min}$ at $200,000 \times \mathrm{g}$ at $4^{\circ} \mathrm{C}$ with the use of a Beckman TLA120.2 rotor to sediment nonbound membranes. The bead pellets were washed five times with PBS. Aliquots of each sample as well as the starting PNS were analyzed by SDS-PAGE and immunoblotting. For detection of Rab5, a rabbit serum (R6) was used with protein A coupled to HRP (Sigma) as a secondary antibody to exclude interference by bead-derived antibodies.

\section{Cell-free Fusion Assay}

For measuring endosome fusion, sets of cells were allowed to internalize biotinylated HRP and avidin, respectively. Upon mixing of PNS, endosome fusion yields a tight complex between avidin and 

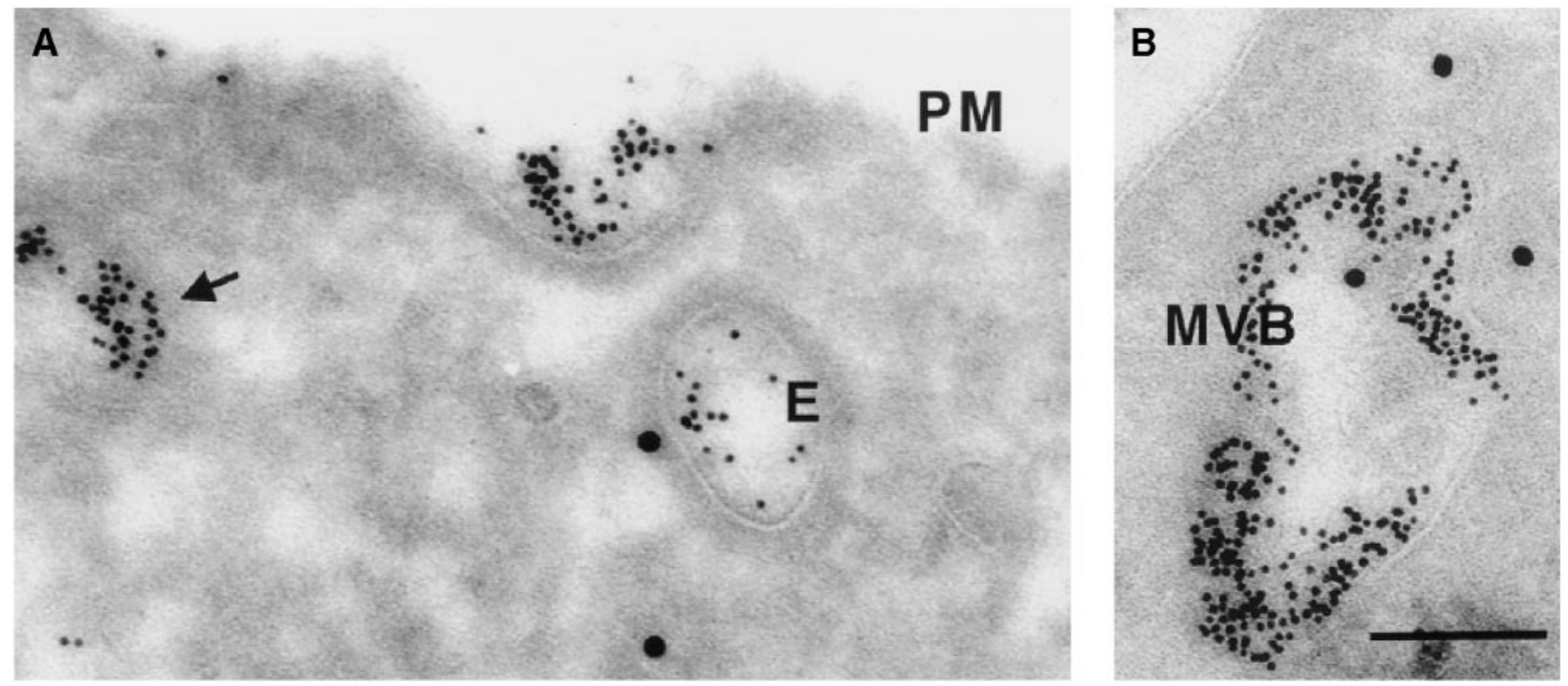

Figure 1. Ultrastructural analysis of endobrevin localization. NRK cells were incubated for $15 \mathrm{~min}$ with BSA-gold (5 $\mathrm{nm}$ ) before fixation and immunolabeling of endobrevin with the use of an affinity-purified rabbit serum and $15 \mathrm{~nm}$ protein A-gold (see MATERIALS AND METHODS). PM, plasma membrane; E, endosome of vacuolar type; MVB, multivesicular body. Note the association of endobrevin with tubulovesicular structures (A, triangle), with endosomal structures of vacuolar type (A, and with endosomes appearing as multivesicular bodies (B). These endobrevin-positive compartments are also positive for the endocytic tracer. Bar, $100 \mathrm{~nm}$.

biotinylated peroxidase, which is quantitated after immunoprecipitation (Gruenberg et al., 1989).

Fluid-phase internalization for labeling of early endosomes of PC12 cells (Greene and Tischler, 1976) was performed as described (Holroyd et al., 1999). For late endosomes, the labeling time was increased to $20 \mathrm{~min}$ followed by five washes for $5 \mathrm{~min}$ with PBS supplemented with $1 \mathrm{mM} \mathrm{MgCl}, 1 \mathrm{mM} \mathrm{CaCl}_{2}$, and $0.5 \% \mathrm{BSA}$ and a 60 -min chase in marker-free medium supplemented with $0.2 \%$ BSA.

The assay for in vitro fusion of early endosomes of PC12 cells was performed as described (Holroyd et al., 1999). The assay for late endosome fusion was identical except that labeling and chase times were changed as described above. The reaction mixtures were increased to $200 \mu \mathrm{l}$ (final volume). Where indicated, both PNS fractions were incubated separately with $6 \mu \mathrm{g}$ of purified Fab fragments or with recombinant protein ( $30 \mu \mathrm{M}$ final concentration) at $37^{\circ} \mathrm{C}$ for $10 \mathrm{~min}$ before combining donor and acceptor PNS fractions for the fusion reaction. For preincubation with cytosol, donor and acceptor PNS were incubated with the appropriate Fab fragments, cytosol, assay buffer, and an ATP-generating system in a final volume of 100 $\mu$ l. Recombinant synaptobrevin (residues 1-96) (Fasshauer et al., 1998a) and endobrevin (residues 1-74) (Fasshauer et al., 1999) were purified as described. All fusion activities were corrected for the activities measured in the absence of ATP (usually $<1 \%$ of the ATP-dependent activities).

\section{In Vitro Assembly of SNARE Proteins}

One microgram of recombinant endobrevin (residues 1-74) (Fasshauer et al., 1999) was preincubated with $20 \mu \mathrm{g}$ of affinitypurified antibodies specific for endobrevin and synaptophysin or with $7 \mu \mathrm{g}$ of the corresponding Fab fragments for 15 min at room temperature. To each sample, $7.5 \mu \mathrm{g}$ of purified binary complex consisting of SNAP-25 and the cytoplasmic region of syntaxin 1 (residues 1-265) (Fasshauer et al., 1999) was added and incubated for $10 \mathrm{~h}$ at $4^{\circ} \mathrm{C}$. As an assay for assembly, the formation of a SDS-resistant complex of endobrevin, syntaxin1, and SNAP-25 was monitored by SDS-PAGE and immunoblotting with the use of the antibody HPC-1 specific for syntaxin-1.

\section{Electron Microscopy}

Immunoelectron microscopy was performed according to the Tokuyasu method (Tokuyasu, 1973; Slot and Geuze, 1985). Nature rat kidney (NRK) cells were incubated for 15 min with BSA coupled to 5-nm gold (BSA-gold) before fixation. The cells were fixed for $2 \mathrm{~h}$ on ice with $2 \%$ paraformaldehyde and $0.2 \%$ glutaraldehyde in $0.1 \mathrm{M}$ K-phosphate buffer, pH 7.3, and embedded into $10 \%$ gelatin. Small blocks were immersed in $2.3 \mathrm{M}$ sucrose overnight for cryoprotection. Ultrathin cryosections were cut from the frozen samples and collected from the diamond knife with a mixture of methylcellulose and sucrose (Liou et al., 1996). The sections were labeled with primary antibodies and detected with protein A-gold purchased from the laboratory of $\mathrm{H}$. Geuze (Utrecht University, Utrecht, the Netherlands). Immunolabeled sections were contrasted with uranyl acetate and embedded into uranyl-methyl cellulose and viewed with a Philips (Eindhoven, the Netherlands) CM120 electron microscope at $80 \mathrm{kV}$.

\section{RESULTS}

\section{Endobrevin/VAMP-8 Is an Abundant Resident of Early and Late Endosomes}

For the characterization of endobrevin, we generated rabbit antisera with the use of bacterially expressed protein. These sera reacted with a single band corresponding to endobrevin in cell extracts (Fasshauer et al., 1999). For further characterization, endobrevin-specific antibodies were affinity-purified with the use of immobilized endobrevin as affinity matrix.

We first determined the distribution of endobrevin within the endocytic pathway from the plasma membrane to lyso- 
Table 1. Quantification of endobrevin in NRK cells

\begin{tabular}{lcrr}
\hline Compartment & $\begin{array}{c}\text { Percent gold } \\
\text { particles }\end{array}$ & $\begin{array}{c}\text { Of these, percent positive } \\
\text { for LIMP II }\end{array}$ & $\begin{array}{c}\text { LIMP II } \\
\text { in NRK }\end{array}$ \\
\hline Golgi/TGN & 12 & 0 & 5 \\
Tubules, vesicles not assigned to TGN or PM & 18 & 14 & 8 \\
Tubulovesicular structures near PM & 26 & 15 & 0 \\
PM & 8 & 71 & 0 \\
MVBs/vacuolar endosomes, ATVs & 29 & 72 \\
Unclassified & 7 & 5 \\
\hline
\end{tabular}

The cells were fixed and labeled for endobrevin and LIMP II as described in MATERIALS AND METHODS. For quantification, 34 sections were analyzed, and a total of 2800 gold particles were counted. PM, plasma membrane; MVBs, multivesicular bodies; ATVs, associated tubules and vesicles.

somes. NRK cells were incubated with BSA-gold as an endocytic marker for $15 \mathrm{~min}$ to label endocytic compartments. The cells were then fixed and analyzed by immunoelectron microscopy of cryosections. Endobrevin labeling was detectable in tubulovesicular structures, small vesicles (Figure 1A, arrow), and endosomes of vacuolar type (Figure 1A) in agreement with Wong et al. (1998b). In addition, endobrevin was found together with BSA-gold in multivesicular bodies (Figure 1B).

To obtain a more detailed overview of the distribution of endobrevin, we performed a quantitative analysis of ultrathin frozen sections labeled for endobrevin. For comparison, the sections were double-labeled for LIMP II, a lysosomal type-3 membrane protein that is also distributed within endosomes (Barriocanal et al., 1986). In NRK cells, the biosynthetic pathway of LIMP II from the TGN to lysosomes is believed to involve endosomes bypassing the plasma membrane (Barriocanal et al., 1986; R. Tikkanen and S. Höning, unpublished data). Endobrevin was abundantly present on tubulovesicular structures near the plasma membrane (Figure 1, Table 1) and on multivesicular bodies and vacuolar endosomes (Figures 1 and 2, Table 1). On the latter compartments, a significant degree of colocalization with LIMP II was observed: $>70 \%$ of the endobrevin-positive structures were also labeled for LIMP II (Table 1). In contrast, only small amounts of LIMP II labeling were detectable on endobrevin-positive tubulovesicular structures near the plasma membrane. When sections labeled for the endogenous MPR46 (Hille-Rehfeld, 1995) and endobrevin were examined, a significant colocalization of endobrevin with MPR46 in TGN-associated structures was observed (Figure 2B, arrowheads). In addition, endobrevin was detectable at the plasma membrane, in agreement with Wong et al. (1998b), and occasionally in clathrin-coated pits (Figure 2C).

Together, these data show that endobrevin is abundantly present on both early and late endosomes. In addition, the presence on the plasma membrane and coated pits indicates that endobrevin may also recycle via the plasma membrane. Endobrevin does not colocalize with the transferrin receptor (our unpublished results; see also Advani et al., 1998), indicating that recycling endosomes are largely devoid of the protein.

To examine the association of endobrevin with early and late endosomes by means of an independent approach, we immunoisolated organelles containing endobrevin and analyzed them for the presence of endosomal markers. For comparison, we also isolated Rab5-containing organelles.
Rab5 is regarded as one of the most specific markers for early endosomes (Novick and Zerial, 1997). For immunoisolation, antibodies for endobrevin and Rab5 were immobilized on methacrylate microbeads (Eupergit C1Z). Immunobeads were incubated with excess amounts of PNS obtained from rat liver. Under these conditions, antigen-containing membranes are only partially depleted, whereas the beads are saturated. This procedure was previously shown to yield organelles of exceptional purity with minimal contamination by other subcellular membranes (Burger et al., 1989; Fischer von Mollard et al., 1994). After isolation of the beads, the unbound membranes were collected by high-speed centrifugation and used as reference. As shown in Figure 3, both Rab5 beads- and endobrevin beads-bound membranes contained their respective antigens. About $50 \%$ of the antigencontaining membranes present in the starting material were bound. Rab5 beads-bound membranes contained endobrevin and endobrevin beads-bound membranes contained Rab5, confirming the association of endobrevin with early endosomes. Interestingly, however, membranes bound to endobrevin beads contained relatively less SCAMP and cellubrevin than membranes bound to Rab5 beads. SCAMP (Brand and Castle, 1993) and cellubrevin (McMahon et al., 1993) recycle between early endosomes and the plasma membrane and are thought to be sorted away from late endosomal/lysosomal compartments. Possibly, there are pools of cellubrevin/SCAMP-containing membranes that contain rab5 but are reduced or devoid of endobrevin, e.g., in the exocytotic limb of the recycling pathway that is highly amplified in liver. In contrast, LIMP II was relatively more enriched on endobrevin beads than on Rab5 beads, confirming the association of endobrevin with LIMP II-containing organelles. Sec61 $\alpha$, a component of the protein translocation complex of the ER (Görlich and Rapoport, 1993), did not bind to the beads, demonstrating the specificity of the immunoisolation procedure. Furthermore, none of the proteins bound to beads containing no antibodies (control beads).

\section{Endobrevin/VAMP-8 Functions in Fusion of Both Early and Late Endosomes}

The data described above show that endobrevin is widely distributed within the endocytic pathway extending from the plasma membrane to early and late endosomes. In addition, smaller pools of endobrevin are also present on TGNassociated membranes, which may reflect either newly syn- 

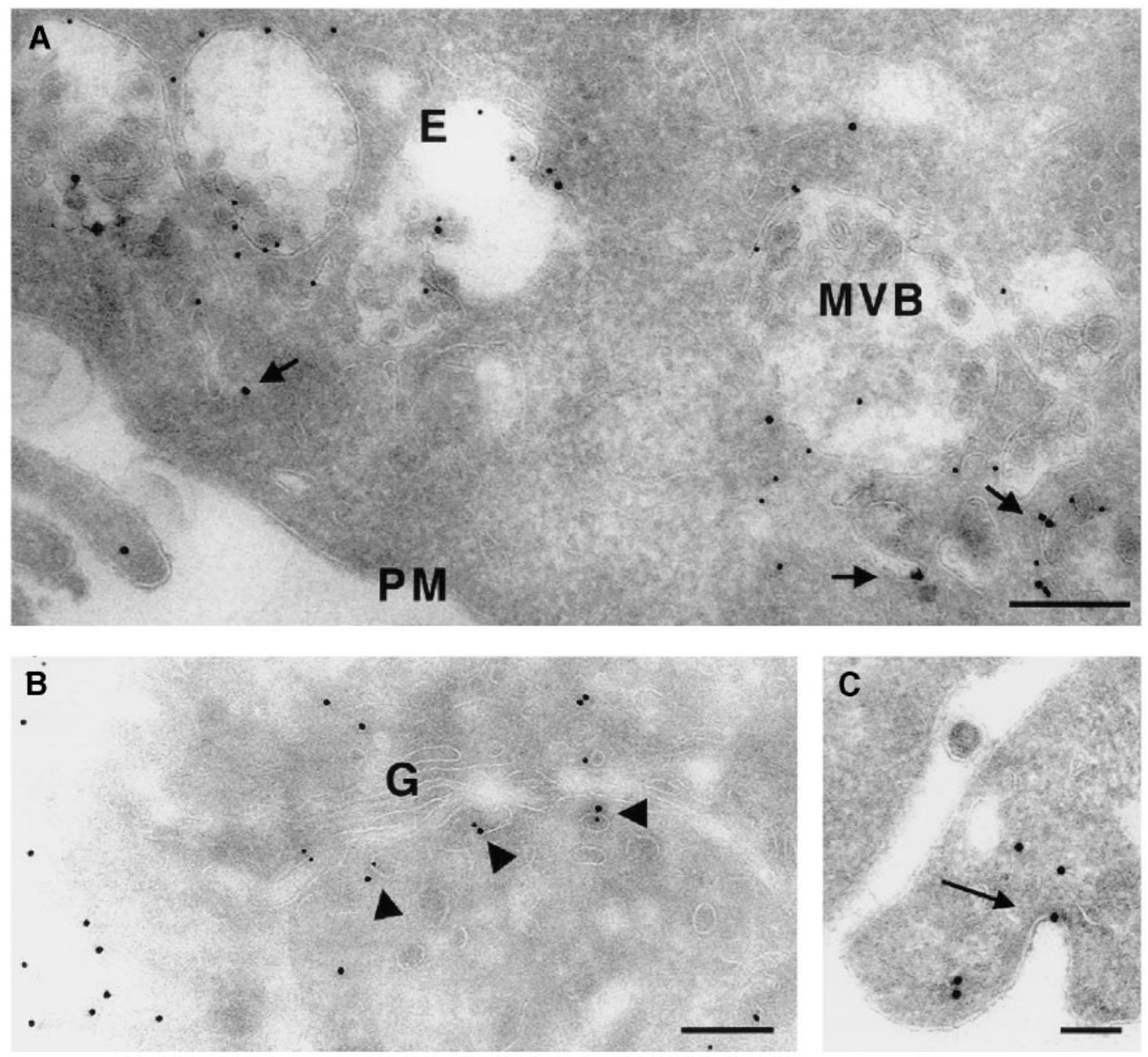

Figure 2. Immunogold localization of endobrevin and LIMP II. Ultrathin cryosections of NRK cells were immunolabeled for endobrevin (15-nm protein A-gold) and either LIMP II (10-nm protein A-gold; A) or the endogenous MPR46 (10-nm protein A-gold; B and C). Endobrevin is detectable in tubulovesicular structures (arrows in A), underneath the plasma membrane (PM), and on endosomal vacuoles (E) and endosomes that appear as multivesicular bodies (MVB), where it often colocalizes with the lysosomal membrane protein LIMP II. We also noted a colocalization of endobrevin with MPR46 in TGN-associated structures (B, arrowheads) and occasionally the appearance in coated pits/vesicles at the plasma membrane (C, arrow). Bars, $100 \mathrm{~nm}$.

thesized protein en route to its destination or a recycling intermediate of the protein. Thus, endobrevin resides on membranes of two recycling pathways that overlap in the endosomal compartment: the first between early endosomes and the plasma membrane, and the second between early and late endosomes/lysosomes, possibly involving the TGN. Each of these pathways involves several distinct fusion and budding steps.

Because the highest concentrations of endobrevin were found on early and late endosomal compartments, we asked whether the protein may be involved in fusion of early and/or late endosomes. These fusion reactions are well characterized now that convenient in vitro assays are available. Both fusion reactions require NSF and ATP and thus are likely to be mediated by SNARE proteins (Robinson et al., 1997). They are distinguished by their preference for the partner membrane, by their requirements for specific Rab proteins (Gorvel et al., 1991; Feng et al., 1995), and probably also by their dependence on Rab-interacting proteins (Stenmark et al., 1995; Horiuchi et al., 1997; Simonsen et al., 1998).

To examine whether endobrevin functions in one of these fusion reactions, we used affinity-purified antibodies as a tool for blocking its SNARE function. Because assembly of cognate SNAREs into core complexes is currently thought to be the decisive step in driving membrane fusion, we first investigated whether the antibodies inhibited the ability of endobrevin to form SDS-resistant complexes. The cognate SNARE partners of endobrevin are not known at present. However, recent evidence has shown that endobrevin can substitute for synaptobrevin in neuronal SNARE complexes. 

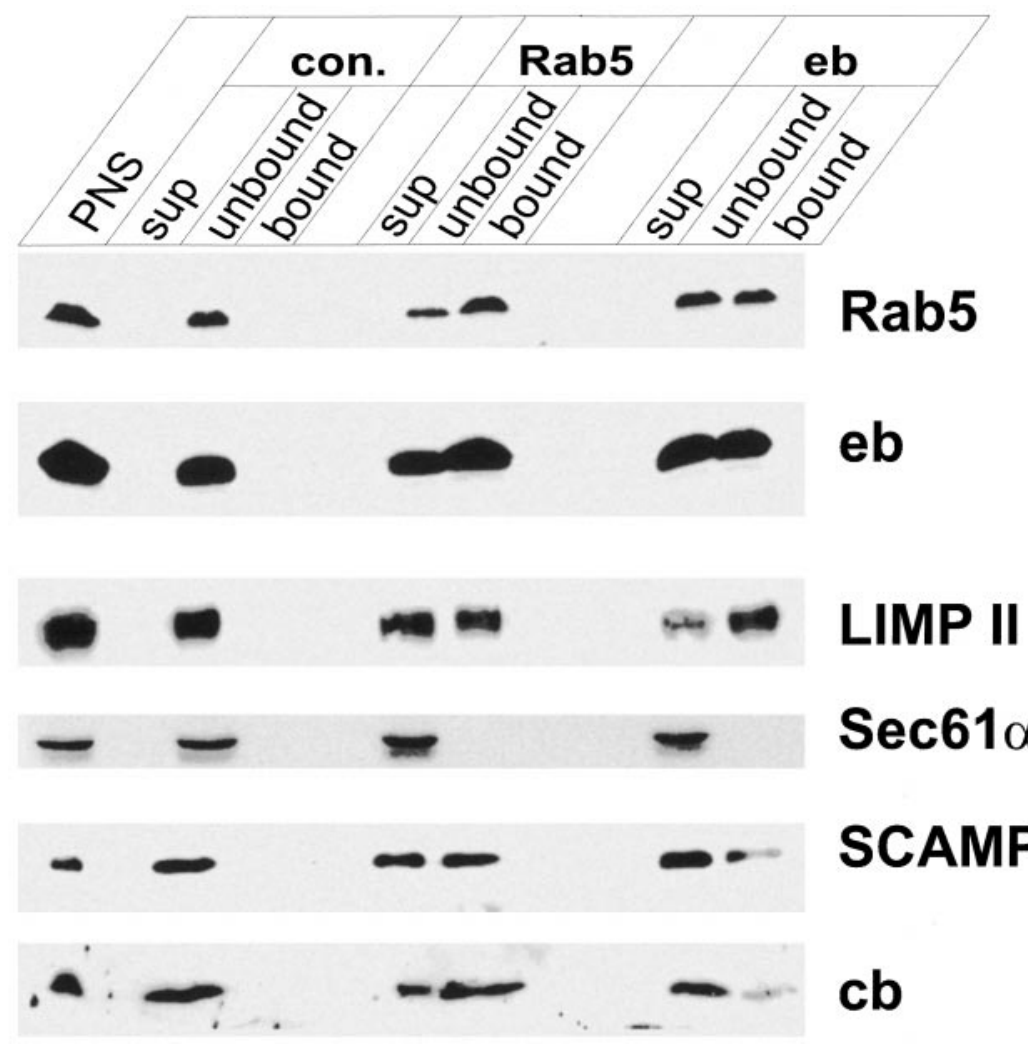

Figure 3. Characterization of organelles immunoisolated with microbeads containing antibodies specific for endobrevin and Rab5. A PNS from rat liver was incubated with small amounts of immunobeads containing covalently bound antibodies specific for Rab5 and endobrevin (eb). Glycine-inactivated beads (con.) served as control for nonspecific binding. The protein composition of the bead-bound membranes (bound) was compared with that of unbound membranes that were sedimented by ultracentrifugation (unbound). Equal proportions of all fractions were analyzed by immunoblotting. PNS, postnuclear supernatant used as starting material; sup, membrane-free supernatant obtained after ultracentrifugation and concentrated by precipitation according to Wessel and Flügge (1984); cb, cellubrevin. PNS, supernatant, and unbound fractions, $10 \mu \mathrm{g}$ of protein/lane; bead-bound material, $\sim 6 \mu \mathrm{l}$ of beads. Note that the membrane protein content of the beads is at least 5- to 10 -fold lower than of the unbound fraction. Also, note that to saturate the beads with organelles, an excess of PNS was used, explaining why none of the antigens was depleted.
The resulting complex is very similar to the neuronal complex with respect to $\alpha$-helical content, SDS and heat resistance, stoichiometry, and susceptibility to disassembly by NSF (Fasshauer et al., 1999; Yang et al., 1999), thus providing a convenient model for endobrevin-SNARE complexes.

Recombinant endobrevin was incubated with affinity-purified antibodies for $15 \mathrm{~min}$ and then combined with recombinant syntaxin 1 and SNAP-25 for the formation of ternary complexes. As a control, parallel incubations were performed with affinity-purified rabbit antibodies for synaptophysin, a major integral membrane protein of synaptic vesicles (Jahn et al., 1985; Wiedenmann and Franke, 1985). As shown in Figure 4 (middle lanes), incubation with endobrevin antibodies completely prevented the formation of SDSresistant complexes. In contrast, complexes were formed when synaptophysin IgG was used or when the antibodies were omitted. Virtually identical results were obtained when Fab fragments instead of intact antibodies were used in the experiment (Figure 4, right lanes).

Next we investigated whether these Fab fragments had an effect on the in vitro fusion of early and late endosomes. For both fusion reactions, in vitro assays are available that were used here with slight modifications. PC12 cells were preloaded by endocytosis with complementary fluid-phase markers with the use of a 5-min pulse for the labeling of early endosomes and a 20 -min pulse followed by a $60-\mathrm{min}$ chase for the labeling of late endosomes. After homogenization, PNS were prepared and combined to initiate the in vitro fusion reaction. Upon fusion, the endocytosed markers form a complex that could be quantified. In some experi- ments, early and late endosomes were separated by sucrose density gradient centrifugation before the fusion assay.

The assays for homotypic fusion of early endosomes and late endosomes showed high ATP-dependent fusion activity (Table 2). In contrast, heterotypic fusion of early and late endosomes was inefficient regardless of whether early or late endosomes were labeled with biotinylated HRP or avidin, respectively. The activities were $\sim 25 \%$ of late endosome

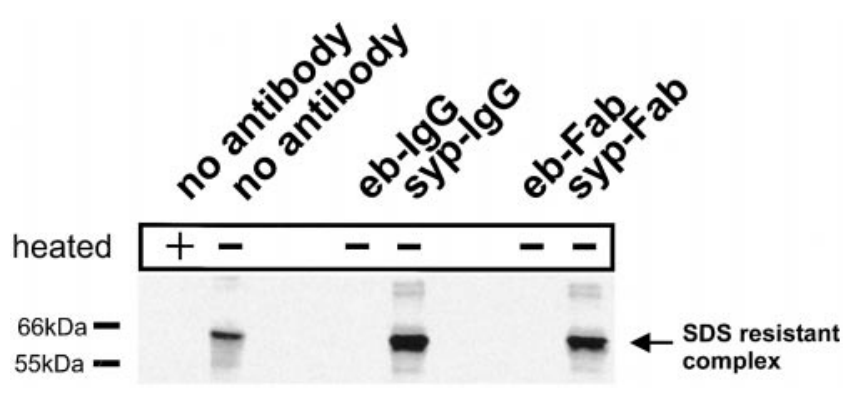

Figure 4. Antibodies specific for endobrevin prevent formation of SDS-resistant SNARE complexes with SNAP-25 and syntaxin 1A. Endobrevin was preincubated with affinity-purified antibodies (IgG) or Fab fragments specific for endobrevin (eb) or synaptophysin (syp) followed by the addition of preassembled SNAP-25/syntaxin 1 complexes. Formation of SDS-resistant complexes was monitored by immunoblotting with the use of an antibody specific for syntaxin 1 . SDS-resistant core complexes are dissociated by boiling (left lane; cf. Hayashi et al., 1994). 
Table 2. ATP-dependent fusion activities (HRP relative units) for early (EE) and late (LE) endosome preparations, labeled with either biotinylated HRP or avidin

\begin{tabular}{lcc}
\hline & EE HRP & LE HRP \\
\hline EE avidin & 322 & 41 \\
LE avidin & 47 & 181 \\
\hline
\end{tabular}

The data are from a representative experiment. Early endosomes were labeled by $5 \mathrm{~min}$ of incubation, and late endosomes were labeled by $20 \mathrm{~min}$ of incubation followed by a 60 -min chase period (see MATERIALS AND METHODS for details).

fusion activity and $15 \%$ of early endosome fusion activity (Table 2). These data show that early endosome and late endosome fusion are distinct fusion events with only minor cross-contamination, in agreement with earlier results (Aniento et al., 1993). For further confirmation, we analyzed the fused membrane vesicles by sucrose density gradient centrifugation. As shown in Figure 5, the fusion products of early and late endosome fusion were well separated. Furthermore, the fusion products of late endosome fusion comigrated with MPR300 but were devoid of cellubrevin, transferrin receptor, and synaptobrevin (our unpublished results), demonstrating that they are free of early endosomes and synaptic vesicles.

When endobrevin-specific antibodies (Fab fragments) were added $10 \mathrm{~min}$ before initiation of the reaction, a significant inhibition of fusion was observed: early endosome fusion was inhibited by $\sim 50 \%$, and late endosome fusion was inhibited by $\sim 60 \%$ (Figure 6 ). Very similar results (early endosomes, 52\% inhibition; late endosomes, $65 \%$ inhibition; data from a single experiment) were obtained when early and late endosomes were prepurified by sucrose gradient centrifugation. As a control, we used antibodies (Fab fragments) specific for synaptophysin, an abundant membrane protein of recycling organelles of neuroendocrine cells, including PC12 cells. These antibodies had no effect on the fusion of late endosomes and only a minor inhibitory effect on early endosome fusion (Figure 6) (see DISCUSSION). Preincubation of anti-endobrevin Fab fragments with stoichiometric amounts of recombinant endobrevin restored fusion activity (our unpublished results), confirming that inhibition of fusion by anti-endobrevin antibodies is due to specific interference with this protein.

It should be noted that inhibition of fusion was not complete. It is possible that antibody binding to endobrevin is inefficient because the protein is complexed to other SNAREs most of the time, forming cis complexes in the endosomal membrane. Because no ATP is present during the preincubation, such complexes would not be disassembled by NSF, resulting in protection of endobrevin from the inactivating antibody until the fusion reaction is initiated. Therefore, Fab fragment preincubation was carried out in the presence of ATP and cytosol as a source for NSF and SNAPs. However, the degree of inhibition did not increase significantly under these conditions, even when the concentrations of NSF and $\alpha$-SNAP were increased by the addition of purified proteins (our unpublished results; see DISCUSSION).
Figure 5. Separation of early and late endosomes by continuous sucrose density gradient centrifugation after completion of the homotypic fusion reaction. Fusion reactions were carried out for early (circles) and late (triangles) endosomes in the presence (closed symbols) or absence (open symbols) of ATP and then loaded in parallel on top of $10-$ $40 \%$ continuous sucrose gradients (see MATERIALS AND METHODS). At the end of the run, fractions of $0.5 \mathrm{ml}$ were collected manually and analyzed by immunoprecipitation for the presence of fusion product. Each immunoprecipitate was assayed for HRP activity with the use of a photometric assay (bottom). The top panel shows the sucrose concentration of each gradient as determined by refractometry (data were obtained from a single experiment).

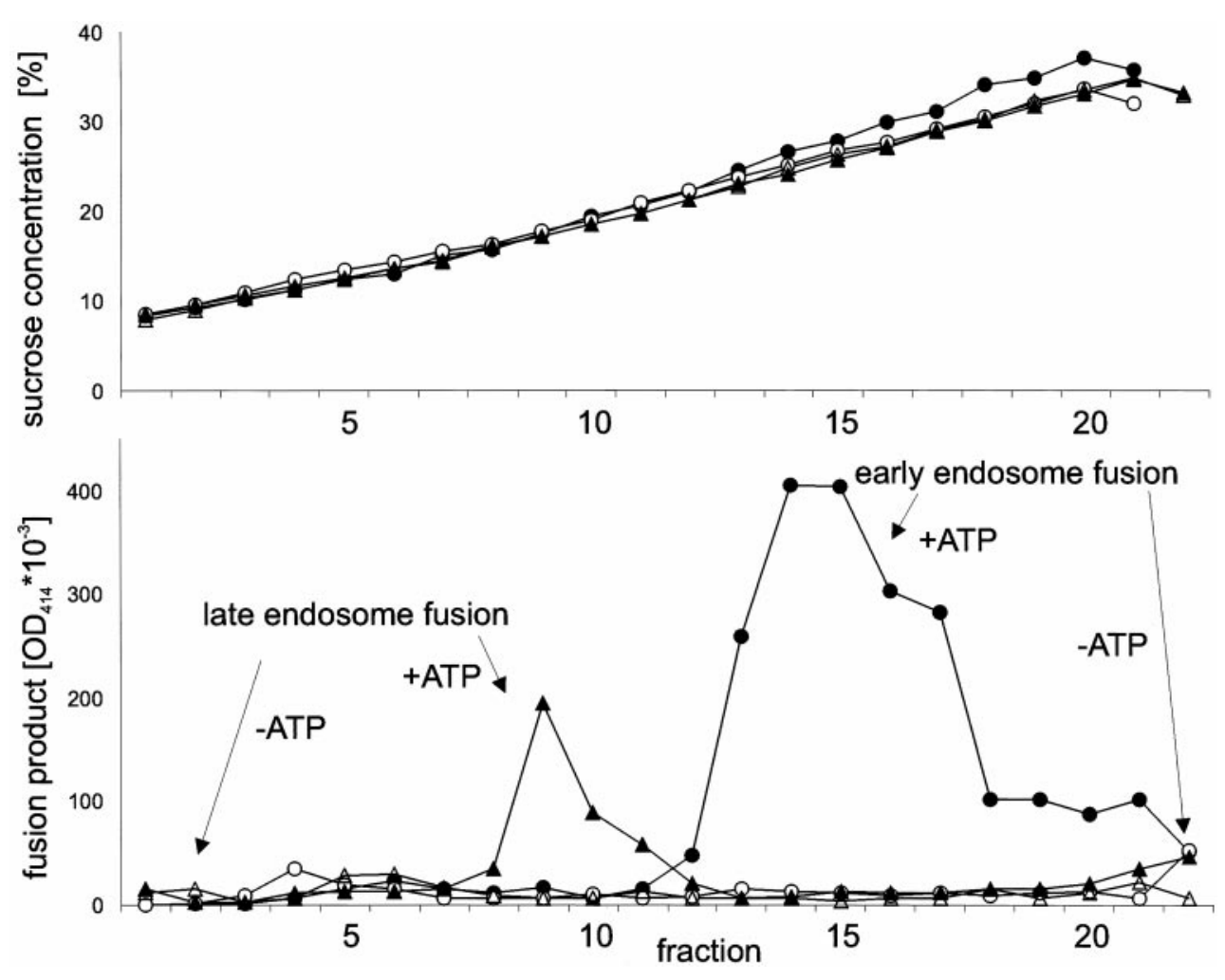



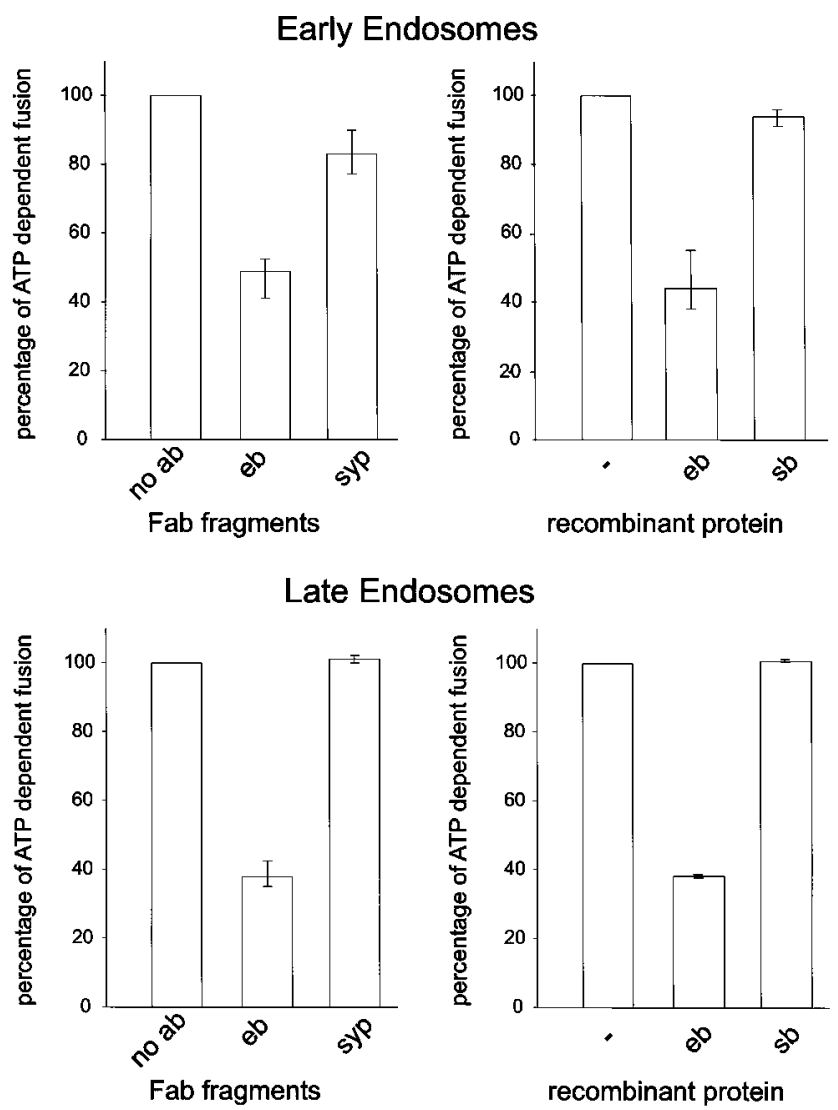

Figure 6. Fusion of early and late endosomes is inhibited by Fab fragments specific for endobrevin or by recombinant endobrevin. Fusion of early and late endosomes derived from PC12 cells was monitored in vitro with the use of a content-mixing assay (see MATERIALS AND METHODS). For preincubation of PNS, $1.2 \mu \mathrm{M}$ Fab fragments specific for endobrevin (eb) or synaptophysin (syp) (left panels) or $30 \mu \mathrm{M}$ soluble recombinant endobrevin or synaptobrevin (sb) (right panels) was used. ATP-dependent fusion activity in the absence of Fab fragments or recombinant protein is defined as $100 \%$. Values are given as means of four independent experiments, and bars indicate the range. Fusion was initiated by mixing donor and acceptor fractions while simultaneously adding ATP and cytosol, followed by $30 \mathrm{~min}$ of incubation.

To confirm the role of endobrevin in early and late endosome fusion by an independent approach, we examined whether the addition of recombinant endobrevin inhibits fusion. As discussed above, the R-SNARE endobrevin is thought to form a complex with cognate SNARE partners during the fusion reaction. Excess amounts of the cytosolic part of endobrevin, therefore, are expected to compete with the endogenous protein. As shown in Figure 6 (right panels), recombinant endobrevin inhibited both fusion reactions as effectively as the Fab fragments. As a control, we added identical amounts of recombinant synaptobrevin, an R-SNARE functioning in exocytosis that is abundantly present on PC12 cell endosomes. No inhibition of either fusion reaction was observed. We conclude from these ex- periments that endobrevin functions as an R-SNARE in the fusion of two distinct intracellular fusion reactions.

\section{DISCUSSION}

In the present study, we have shown that endobrevin, an R-SNARE with a widespread distribution throughout the endocytic pathway, functions in the homotypic fusion of early and late endosomes.

Two independent approaches were chosen to investigate the role of endobrevin in endosome fusion, giving similar results. Antibodies are frequently used to inhibit the function of a protein in membrane fusion (McBride et al., 1999). However, it needs to be borne in mind that inhibition of fusion may be caused by the presence of bulky IgG molecules on the fusing membranes, thereby preventing membrane contact. An appropriate control for such indirect inhibition involves the use of antibodies specific for an abundant membrane protein of endosomes that is not involved in membrane fusion. Therefore, we chose PC12 cells, which, unlike neurons, abundantly express endobrevin, allowing us to use probes specific for synaptic vesicle proteins such as synaptophysin. Synaptophysin is highly abundant not only on exocytotic vesicles but also on early endosomes (Holroyd et al., 1999), providing a convenient control, at least for the fusion of early endosomes. Interestingly, intact IgG specific for synaptophysin (in contrast to Fab fragments) potently inhibited this fusion step (our unpublished observations), confirming that inhibition of in vitro fusion reactions may indeed be caused by such indirect effects.

It is noteworthy that the fusion reactions are inhibited by the addition of exogenous soluble endobrevin but not of synaptobrevin. In vitro, both endobrevin and synaptobrevin form SNARE complexes of similar structural properties with the same partner proteins (Fasshauer et al., 1999; Yang et al., 1999). The selectivity observed here supports the view that there must be at least some preference for the cognate SNARE. Preliminary observations suggest that this specificity may be overcome by the use of excessive amounts of synaptobrevin (our unpublished observations).

It remains to be established whether endobrevin is the only R-SNARE required for early and late endosome fusion or whether other, hitherto unidentified, R-SNAREs operate alongside endobrevin. The latter possibility is suggested by the observation that some fusion persisted in the presence of our antibodies, although these antibodies completely prevented assembly of endobrevin with Q-SNAREs in an in vitro assembly assay. Interestingly, neurons do not express endobrevin (Advani et al., 1998; our unpublished observations), although fusion of early endosomes is thought to be involved in both synaptic and extrasynaptic trafficking pathways (Jessell and Kandel, 1993), suggesting the involvement of additional R-SNAREs. Cellubrevin, an abundant R-SNARE recycling between the plasma membrane and early endosomes, is not involved in early endosome fusion. Unlike endobrevin (our unpublished observations), cellubrevin is efficiently cleaved by clostridial neurotoxins, whereas endosome fusion is not affected by toxin treatment (Link et al., 1993; Holroyd et al. 1999). Rather, cellubrevin appears to function in the fusion of transport vesicles with the plasma membrane (Galli et al., 1994). Recently, VAMP-7, which is localized to late endosomes in addition to the TGN 
and transport vesicles, has been suggested to operate in membrane traffic from the late endosome to the lysosome (Advani et al., 1999). Other candidates include VAMP-4, which was localized to endosomes (Steegmaier et al., 1999).

It is not yet known with which Q-SNAREs endobrevin interacts and whether its Q-SNARE partners are identical in the two fusion reactions. Recently, syntaxin 7 (Wong et al., 1998a) and syntaxin 12/13 (Advani et al., 1998; Tang et al., 1998) (syntaxin 12 and syntaxin 13 probably represent incomplete sequences of the same protein) have been localized to early endosomes, making them candidates for such QSNARE partners. Furthermore, syntaxin 13 was recently suggested to be involved in the fusion of early endosomes (McBride et al., 1999). In preliminary experiments, however, we were unable to coprecipitate endobrevin with syntaxin 13 from tissue extracts (our unpublished observations). Also, it remains to be established whether a relative of SNAP-25, e.g., SNAP-23 (Ravichandran et al., 1996) or the recently identified SNAP-29/GS-32 (Steegmaier et al., 1998; Wong et al., 1999), is participating in endobrevin-SNARE complexes.

In conclusion, our data lend strong support to the view that the assembly of specific sets of SNARE proteins is involved in many, perhaps all, intracellular fusion steps. The ongoing characterization of new mammalian SNAREs raises the hope that the SNARE partners of endobrevin as well as the SNARE complexes functioning in other intracellular fusion steps will soon be identified.

\section{ACKNOWLEDGMENTS}

We are greatly indebted to Dr. C. Barnstable, Dr. E. Hartmann, Dr. A. Hille-Rehfeld, and Dr. Y. Tenaka for their kind gifts of antibodies, and to Dr. D. Fasshauer and M. Margittai for the gift of recombinant SNAP-25, syntaxin1A, and synaptobrevin, respectively. We thank Dr. D. Bruns, Dr. D. Fasshauer, P. Holroyd, M. Margittai, S. Pabst, and $\mathrm{B}$. Rammner for critically reading the manuscript and for fruitful discussions. This work was supported by grants from the Deutsche Forschungsgemeinschaft (SFB 532, TP A5, TP B6, TP Z2).

\section{REFERENCES}

Advani, R.J., Bae, H.R., Bock, J.B., Chao, D.S., Doung, Y.C., Prekeris, R., Yoo, J.S., and Scheller, R.H. (1998). Seven novel mammalian SNARE proteins localize to distinct membrane compartments. J. Biol. Chem. 273, 10317-10324.

Advani, R.J., Yang, B., Prekeris, R., Lee, K.C., Klumperman, J., and Scheller, R.H. (1999). VAMP-7 mediates vesicular transport from endosomes to lysosomes. J. Cell Biol. 146, 765-776.

Aniento, F., Emans, N., Griffiths, G., and Gruenberg, J. (1993). Cytoplasmic dynein-dependent vesicular transport from early to late endosomes. J. Cell Biol. 123, 1373-1387.

Annaert, W.G., Becker, B., Kistner, U., Reth, M., and Jahn, R. (1997). Export of cellubrevin from the endoplasmic reticulum is controlled by BAP31. J. Cell Biol. 139, 1397-1410.

Barnstable, C.J., Hofstein, R., and Akagawa, K. (1985). A marker of early amacrine cell development in rat retina. Brain Res. 352, 286-290.

Barriocanal, J.G., Bonifacino, J.S., Yuan, L., and Sandoval, I.V. (1986). Biosynthesis, glycosylation, movement through the Golgi system, and transport to lysosomes by an N-linked carbohydrate-independent mechanism of three lysosomal integral membrane proteins. J. Biol. Chem. 261, 16755-16763.

Bock, J.B., and Scheller, R.H. (1997). Protein transport: a fusion of new ideas. Nature 387, 133-135.
Brand, S.H., and Castle, J.D. (1993). SCAMP 37, a new marker within the general cell surface recycling system. EMBO J. 12, 3753-3761.

Burger, P.M., Mehl, E., Cameron, P.L., Maycox, P.R., Baumert, M., Lottspeich, F., De Camilli, P., and Jahn, R. (1989). Synaptic vesicles immunoisolated from rat cerebral cortex contain high levels of glutamate. Neuron 3, 715-720.

Dascher, C., and Balch, W.E. (1996). Mammalian Sly1 regulates syntaxin 5 function in endoplasmic reticulum to Golgi transport. J. Biol. Chem. 271, 15866-15869.

Dascher, C., Matteson, J., and Balch, W.E. (1994). Syntaxin 5 regulates endoplasmic reticulum to Golgi transport. J. Biol. Chem. 269, 29363-29366.

Fasshauer, D., Antonin, W., Margittai, M., Pabst, S., and Jahn, R. (1999). Mixed and non-cognate SNARE complexes: characterization of assembly and biophysical properties. J. Biol. Chem. 274, 15440-15446.

Fasshauer, D., Eliason, W.K., Brunger, A.T., and Jahn, R. (1998a). Identification of a minimal core of the synaptic SNARE complex sufficient for reversible assembly and disassembly. Biochemistry 37, 10354-10362.

Fasshauer, D., Sutton, R.B., Brunger, A.T., and Jahn, R. (1998b). Conserved structural features of the synaptic fusion complex: SNARE proteins reclassified as Q- and R-SNAREs. Proc. Natl. Acad. Sci. USA 95, 15781-15786.

Feng, Y., Press, B., and Wandinger-Ness, A. (1995). Rab 7: an important regulator of late endocytic membrane traffic. J. Cell Biol. 131, $1435-1452$

Fischer von Mollard, G., Stahl, B., Walch-Solimena, C., Takei, K., Daniels, L., Khoklatchev, A., De Camilli, P., Sudhof, T.C., and Jahn, R. (1994). Localization of Rab5 to synaptic vesicles identifies endosomal intermediate in synaptic vesicle recycling pathway. Eur. J. Cell Biol. 65, 319-326.

Galli, T., Chilcote, T., Mundigl, O., Binz, T., Niemann, H., and De Camilli, P. (1994). Tetanus toxin-mediated cleavage of cellubrevin impairs exocytosis of transferrin receptor-containing vesicles in CHO cells. J. Cell Biol. 125, 1015-1024.

Görlich, D., Prehn, S., Hartmann, E., Kalies, K.U., and Rapoport, T.A. (1992). A mammalian homolog of SEC61p and SECYp is associated with ribosomes and nascent polypeptides during translocation. Cell 71, 489-503.

Görlich, D., and Rapoport, T.A. (1993). Protein translocation into proteoliposomes reconstituted from purified components of the endoplasmic reticulum membrane. Cell 75, 615-630.

Gorvel, J.P., Chavrier, P., Zerial, M., and Gruenberg, J. (1991). rab5 controls early endosome fusion in vitro. Cell 64, 915-925.

Götte, M., and von Mollard, G.F. (1998). A new beat for the SNARE drum. Trends Cell Biol. 8, 215-218.

Greene, L.A., and Tischler, A.S. (1976). Establishment of a noradrenergic clonal line of rat adrenal pheochromocytoma cells which respond to nerve growth factor. Proc. Natl. Acad. Sci. USA 73, $2424-2428$.

Gruenberg, J., Griffiths, G., and Howell, K.E. (1989). Characterization of the early endosome and putative endocytic carrier vesicles in vivo and with an assay of vesicle fusion in vitro. J. Cell Biol. 108, 1301-1316.

Hanson, P.I., Heuser, J.E., and Jahn, R. (1997). Neurotransmitter release: four years of SNARE complexes. Curr. Opin. Neurobiol. 7, 310-315.

Hayashi, T., McMahon, H., Yamasaki, S., Binz, T., Hata, Y., Sudhof, T.C., and Niemann, H. (1994). Synaptic vesicle membrane fusion complex: action of clostridial neurotoxins on assembly. EMBO J. 13, 5051-5061. 
Hille-Rehfeld, A. (1995). Mannose 6-phosphate receptors in sorting and transport of lysosomal enzymes. Biochim. Biophys. Acta 1241, 177-194.

Holroyd, C., Kistner, U., Annaert, W., and Jahn, R. (1999). Fusion of endosomes involved in synaptic vesicle recycling. Mol. Biol. Cell 10, 3035-3044

Horiuchi, H., et al. (1997). A novel Rab5 GDP/GTP exchange factor complexed to Rabaptin-5 links nucleotide exchange to effector recruitment and function. Cell 90, 1149-1159.

Jahn, R., Schiebler, W., Ouimet, C., and Greengard, P. (1985). A 38,000 -dalton membrane protein (p38) present in synaptic vesicles. Proc. Natl. Acad. Sci. USA 82, 4137-4141.

Jahn, R., and Südhof, T.C. (1999). Membrane fusion and exocytosis. Annu. Rev. Biochem. 68, 863-911.

Jessell, T.M., and Kandel, E.R. (1993). Synaptic transmission: a bidirectional and self-modifiable form of cell-cell communication. Cell 72, 1-30.

Link, E., McMahon, H., Fischer von Mollard, G., Yamasaki, S., Niemann, H., Sudhof, T.C., and Jahn, R. (1993). Cleavage of cellubrevin by tetanus toxin does not affect fusion of early endosomes. J. Biol. Chem. 268, 18423-18426.

Liou, W., Geuze, H.J., and Slot, J.W. (1996). Improving structural integrity of cryosections for immunogold labeling. Histochem. Cell Biol. 106, 41-58.

McBride, H.M., Rybin, V., Murphy, C., Giner, A., Teasdale, R., and Zerial, M. (1999). Oligomeric complexes link Rab5 effectors with NSF and drive membrane fusion via interactions between EEA1 and syntaxin 13. Cell 98, 377-386.

McMahon, H.T., Ushkaryov, Y.A., Edelmann, L., Link, E., Binz, T., Niemann, H., Jahn, R., and Sudhof, T.C. (1993). Cellubrevin is a ubiquitous tetanus-toxin substrate homologous to a putative synaptic vesicle fusion protein. Nature 364, 346-349.

Navone, F., Jahn, R., Di Gioia, G., Stukenbrok, H., Greengard, P., and De Camilli, P. (1986). Protein p38: an integral membrane protein specific for small vesicles of neurons and neuroendocrine cells. J. Cell Biol. 103, 2511-2527.

Nichols, B.J., Ungermann, C., Pelham, H.R., Wickner, W.T., and Haas, A. (1997). Homotypic vacuolar fusion mediated by t- and v-SNAREs. Nature 387, 199-202.

Novick, P., and Zerial, M. (1997). The diversity of Rab proteins in vesicle transport. Curr. Opin. Cell Biol. 9, 496-504.

Rabouille, C., Kondo, H., Newman, R., Hui, N., Freemont, P., and Warren, G. (1998). Syntaxin 5 is a common component of the NSFand p97-mediated reassembly pathways of Golgi cisternae from mitotic Golgi fragments in vitro. Cell 92, 603-610.

Ravichandran, V., Chawla, A., and Roche, P.A. (1996). Identification of a novel syntaxin- and synaptobrevin/VAMP-binding protein, SNAP-23, expressed in non-neuronal tissues. J. Biol. Chem. 271, 13300-13303.

Robinson, L.J., Aniento, F., and Gruenberg, J. (1997). NSF is required for transport from early to late endosomes. J. Cell Sci. 110, 2079-2087.

Rothman, J.E. (1994). Mechanisms of intracellular protein transport. Nature 372, 55-63.
Simonsen, A., Lippe, R., Christoforidis, S., Gaullier, J.M., Brech, A., Callaghan, J., Toh, B.H., Murphy, C., Zerial, M., and Stenmark, H. (1998). EEA1 links PI(3)K function to Rab5 regulation of endosome fusion. Nature 394, 494-498

Slot, J.W., and Geuze, H.J. (1985). A new method of preparing gold probes for multiple-labeling cytochemistry. Eur. J. Cell Biol. 38, 87-93.

Söllner, T., Whiteheart, S.W., Brunner, M., Erdjument-Bromage, H., Geromanos, S., Tempst, P., and Rothman, J.E. (1993). SNAP receptors implicated in vesicle targeting and fusion. Nature 362, 318-324.

Spang, A., and Schekman, R. (1998). Reconstitution of retrograde transport from the Golgi to the ER in vitro. J. Cell Biol. 143, 589-599.

Steegmaier, M., Klumperman, J., Foletti, D.L., Yoo, J.S., and Scheller, R.H. (1999). Vesicle-associated membrane protein 4 is implicated in trans-Golgi network vesicle trafficking. Mol. Biol. Cell 10, 1957-1972.

Steegmaier, M., Yang, B., Yoo, J.S., Huang, B., Shen, M., Yu, S., Luo, Y., and Scheller, R.H. (1998). Three novel proteins of the syntaxin/ SNAP-25 family. J. Biol. Chem. 273, 34171-34179.

Stenmark, H., Vitale, G., Ullrich, O., and Zerial, M. (1995). Rabaptin-5 is a direct effector of the small GTPase Rab5 in endocytic membrane fusion. Cell 83, 423-432.

Sutton, R.B., Fasshauer, D., Jahn, R., and Brunger, A.T. (1998). Crystal structure of a SNARE complex involved in synaptic exocytosis at $2.4 \AA$. Nature 395, 347-353.

Tang, B.L., Tan, A.E., Lim, L.K., Lee, S.S., Low, D.Y., and Hong, W. (1998). Syntaxin 12, a member of the syntaxin family localized to the endosome. J. Biol. Chem. 273, 6944-6950.

Tokuyasu, K.T. (1973). A technique for ultracryotomy of cell suspensions and tissues. J Cell Biol. 57, 551-565.

Ungermann, C., and Wickner, W. (1998). Vam7p, a vacuolar SNAP-25 homolog, is required for SNARE complex integrity and vacuole docking and fusion. EMBO J. 17, 3269-3276.

Weimbs, T., Mostov, K., Low, S.H., and Hofmann, K. (1998). A model for structural similarity between different SNARE complexes based on sequence relationships. Trends Cell Biol. 8, 260-262.

Wessel, D., and Flügge, U.I. (1984). A method for the quantitative recovery of protein in dilute solution in the presence of detergents and lipids. Anal. Biochem. 138, 141-143.

Wiedenmann, B., and Franke, W.W. (1985). Identification and localization of synaptophysin, an integral membrane glycoprotein of $\mathrm{Mr}$ 38,000 characteristic of presynaptic vesicles. Cell 41, 1017-1028.

Wong, S.H., Xu, Y., Zhang, T., Griffiths, G., Lowe, S.L., Subramaniam, V.N., Seow, K.T., and Hong, W. (1999). GS32, a novel Golgi SNARE of $32 \mathrm{kDa}$, interacts preferentially with syntaxin 6. Mol. Biol. Cell 10, 119-134.

Wong, S.H., Xu, Y., Zhang, T., and Hong, W. (1998a). Syntaxin 7, a novel syntaxin member associated with the early endosomal compartment. J. Biol. Chem. 273, 375-380.

Wong, S.H., Zhang, T., Xu, Y., Subramaniam, V.N., Griffiths, G., and Hong, W. (1998b). Endobrevin, a novel synaptobrevin/VAMP-like protein preferentially associated with the early endosome. Mol. Biol. Cell 9, 1549-1563.

Yang, B., Gonzalez, L., Jr., Prekeris, R., Steegmaier, M., Advani, R.J., and Scheller, R.H. (1999). SNARE interactions are not selective: implications for membrane fusion specificity. J. Biol. Chem. 274, 5649-5653. 